\title{
COLLABORATIVE NOISE MAPPING USING SMARTPHONE
}

\author{
Rakesh Dubey ${ }^{1, *}$, Shruti Bharadwaj ${ }^{1}$, MD Iltaf Zafar ${ }^{1}$, Vinamra Bhushan Sharma ${ }^{1}$, Susham Biswas ${ }^{1}$ \\ ${ }^{1}$ Dept. of Chemical Engineering and Engineering Sciences, Rajiv Gandhi Institute of Petroleum Technology Jais, Amethi, \\ Uttar Pradesh, INDIA- (pgi19001, pgi17001, mzafar, pgi19002, susham)@rgipt.ac.in
}

KEYWORDS: GIS, GPS, Noise Mapping, Noise Modeling, Smart Phones, Road Traffic Noise

\begin{abstract}
:
Noise pollution is considered to be one of the most prevalent environmental challenges affecting human health. Noise pollution is increasing in cities needing techniques to monitor and predict the noise. The monitoring of traffic noise levels in different parts of the cities at different times has become very difficult due to logistic constraints. It is thus required to measure the noise levels at certain strategic locations, such as, near the noise sources (e.g., roads), and then to utilize it to predict the noise levels at surrounding locations. The challenge of monitoring the noise near several road crossings in a city can be reduced using a smartphone-based noise monitoring technique. However, the prediction of noise levels and showcase it as maps require terrain data, noise data, and noise prediction models. The requirement of terrain data can be met using open-source terrain data, from which various terrain parameters can be extracted and integrated with a standard prediction model on the web platform to predict the noise map for an area. Smartphone-based noise monitoring and its subsequent mapping can be a very popular and effective option, which uses a crowdsourcing approach. The entire methodology is tried to be applied over Lucknow city in India. Noise levels are monitored at three different slots, daily, over 14 road crossings using the smartphone-based app. Further, collected noise levels were calibrated against standard noise meter to ascertain accurate noise levels for these locations. Thereafter, three categories of noise environments are chosen from it and mapped using open-source satellite images and standard noise models, over the web on the GIS platform. The predicted noise levels on the maps were verified with the recorded noise data from similar locations using standard noise meter. For these three crossings at different times the predictions are found to be accurate within $\pm 4.5 \mathrm{~dB}$.
\end{abstract}

\section{INTRODUCTION}

Noise is a general problem that carries important health concerns. We are moving towards modernization with the advancement in technology. Better-suited technologies make our life easy and convenient (Tandel and Sonaviya 2018). However, newer technologies or progress are also associated with health hazards. The health issues are more prominent in developing countries as compared with developed countries. India is trying to diminish the effect of noise to a manageable level by monitoring, analyzing, and warning the public of its ill effect. The problem of noise is prominent in cities. This is more because of the traffic noise. Other than traffic noise, the activities in market places, buzzing of loudspeakers, and ancillary activities add noise hazards in cities. Further in India, land use pattern is mixed, thus silence zone (hospital, school, residential area) are next to the busy road or market places.

Traffic noises are associated with health hazards such as cardiovascular diseases, hearing disorders, high blood pressure, annoyances, etc. Hearing a loud noise in city ambiance during day and night impacts the physiological changes like hearing impairment. It also can have some psychological hazards associated with it. In a recent study in Spain (Münzel et al. 2018), a report states that noise pollution has become so dominant that it has crossed the environmental risk factor of air pollution. Also, its effect is often overlooked despite being linked to an increased risk of early death. Incidentally, there are other factors, e.g., air pollution, hereditary disease, social lifestyle diseases (overconsumption of alcohol or smoking) which can also make damages to health, showing similar symptoms.
So, it becomes challenging to find the real causative factors for the above health concerns. The main strategies that have to be made to combat such issues require accurate monitoring of traffic noise, measurement of traffic noise for large city areas, determination of noise hazards for different parts of cities, and estimation of health issues taking the inputs from people of the city. The government needs to aware of the public frequently with proper campaigns.

In that context of proper management of the traffic noise, it is required to comprehensively monitor and /or map the noise levels of different parts of the cities. It is not feasible to measure the noise levels for large areas of any city all the time. To do it, a large number of expensive noise meters or sound pressure level meters will be required. In the absence of such big hardware resources, it becomes necessary to measure the noise levels at certain strategic locations, e.g., near noise corridors of roads and then model or map it to its nearby areas. Further, the collection of accurate noise data near noise sources also becomes a challenge due to the requirement of a noise meter. It is required to have a low cost, efficient, and convenient mapping solution. Thus, smartphones giving noise maps are thought of. However, the smartphone-based technique will have a problem of accuracy for the collected noise data, which is required to be improved. Smartphone-based technique if acceptable will have the advantages of collecting the data from large areas taking the input from the dense population in cities. The efficient crowdsourcing technique over the internet provides a big opportunity to utilize the monitored data to map the noise levels for cities. Noise mapping requires noise data, terrain data, and noise model. Once the above data are managed it can be mapped over the ArcGIS platform.

\footnotetext{
* Corresponding author
} 


\section{LITERATURE REVIEW}

\subsection{GIS-based Noise Mapping}

This GIS-based mapping has boomed in the recent past with its application in merely all the fields. It has also has come up in broadening of access to spatial data. In the current scenario, in respect of noise pollution that can have a serious health concern, GIS-based noise mapping has become a very useful application. It offers numerically simulated data with software specially developed for environmental noise prediction applications. It uses the principles of acoustics from different sources (Bocher et al. 2019). Here, the GIS-based calculation is done in the background with proper visualization and analyzation embedded in the spatial domain. With the above background, the authors tried to carry out the study in Lucknow city. It was required to ascertain when, where, or how an efficient mapping can be carried out. The monitoring of noise data, the use of spatial data, and modeling to map the noise over arc GIS platforms are required to be ascertained. The projected noise maps once prepared are required to be compared spatially for relative impacts. Further, this map was also required to be linked with people's concerns for health due to noise pollution. In this way, people in a different place, a time slot can experience and participate in computing out the noise pollution at their end.

A coherent study was carried out in the city of Bengal where the time-space and noise distribution for traffic over the roads and the nearby building were determined using the SPL (Sound Pressure Level, Meter) and was mapped on web-platform using the ArcGIS (Banerjee et al. 2008). It showed that the noise level in all the studied locations exceeds the limit prescribed by CPCB (Central Pollution Control Board, India). In the same way on the same experimental setup the study was carried out in the city of Amman in Al-Shaheed street, where the data was recorded using the SPL and mapped using the ArcGIS and the recorded data was computed over the map. Lequivalent values determined there varied from $60 \mathrm{~dB}$ (A) to $77 \mathrm{~dB}(\mathrm{~A}) .25 .5 \%$ reduction in light vehicles has resulted in $2.5 \%$ of less noise levels in some cases (Mofeed, Imam, and Jamrah 2013). On the other hand, in the city of Nigeria, similar data were recorded and showcased in the GIS platform.

Noise recordings were done for three slots in a day - morning, noon, and evening. On the GIS platform, different IDW interpolations were made for the map and the different range was set according to the WHO standard for the annoyances with the help of spatial interpolations. The lowest daily average of noise ranged between $67.2 \mathrm{~dB}(\mathrm{~A})$ and $76.7 \mathrm{~dB}(\mathrm{~A})$ across all the land uses(Akintuyi, Raji, and Wunude 2014). Among the related articles only one of the two studies has shown the exposure of the noise effect using the audiometric test. Other than this some have used the questionnaire survey to ascertain the noise ambiance in an area. However, these surveys gave a very average idea of noise for the area, as are limited to the specific zone due to it's the inability to consider the terrain parameters (Biswas and Lohani 2008), and other circumstances that change over time and place. Generally, these study of noise pollution or their mapping approaches indicated

- Different sorts of models were used in the studies. Also, the questionnaires used were quite different from each other as they didn't have followed the same set of questionnaires to draw any uniformity.

- The sample sizes were different so the comparison of the data from any referenced base data required extra regression using a meta-analysis of these articles. Around $76 \%$ of the researchers have used the statistical approach.

- Out of the reviewed article, $37 \%$ of the data have used the questionnaire survey and were distinct from each other and mapped roughly.

- $\quad$ Noise pollutions in cities/towns of India were different and show varied health hazards.

\subsection{Web-based Noise Mapping}

The primary goal of the web-based GIS platform is to provide an easy and convenient way to derive the map on the ArcGIS and can be easily be seen over the web by the users. Here, it is assumed that users are having access to the internet. Different types of data or parameters are uploaded on the web which is spatially referenced and mapped over the internet. Further, it offered the opportunity to share files that can easily be monitored over the web. ArcGIS merely comprised of four key parts, firstly the geographically based model for modeling; secondly, it comprised of various components for storing and managing geographic information; thirdly it offered the in inbuilt applications for creating, editing, mapping, analyzing and disseminating geographic information; and at last the collection of web services that provided the content and capabilities to networked software clients (Maguire 2016). Parts of the ArcGIS software system can be developed on mobile devices, laptops, and desktop computers and servers. ArcGIS made it easy to analyze field data, both on the desktop and the cloud. We can symbolize and classify our data, perform spatial analysis. We can also present our results online as live web maps, embed our maps in web pages, and use them to create story maps. Esri has provided the platform from which all the members can easily access and use the geographical information within a collaborative environment. One of the key ideas of the Esri Geospatial Cloud is the Web GIS pattern, that, all members of an organization can easily access and use for geographic information within a collaborative environment. It provided a convenient interface that a non-technical person who doesn't have much of the knowledge can also leverage on the GIS platform. It also made them usable and more accessible. It provided a platform for integration, promoting crossorganizational collaboration, and enabling decision-making (Esri 2018).

\subsection{Collaborative mapping through crowdsourcing using smartphones}

In the crowd-based noise mapping technique, the data is been inculcated over the web from different sources working over the region with the same platform of the same database and they all are connected with the same interface. In this, the data is being stimulated in a particular range where the correctly collected data goes in the sink zone and rest goes in the trash which is of no use. Later this collected data is being monitored and mapped over the web using the ArcGIS and later on it can be seen using the smartphone-based android app for GIS mapping. Also, in this approach, the general public can participate in collaborative mapping and compare the results over the web. The app-based smartphone with inbuilt GPS when used can help to measure the extent of spatial dispersion of any parameters. Thus, this contribution from each individual is been shared in the form of geo-localized measurement and then can be displayed with personally performed annotation to produce any map for different users. Contribution and data collation at different stages will make the data more precise close to the true value. Collection at each level by each individual is also being 
monitored/recorded and is possible to be taken into account by the app for obtaining and retaining the personal interest or customized maps (Bocher; et al. 2015).

\section{RESEARCH GAP}

Noise mapping required the detailed noise data of a large area. Researchers primarily used limited noise data, which are collected using costly Noise Meter. Further, it becomes a challenge to collect accurate noise data at different times in a day, over weeks, and different seasons. Furthermore, the mapping also required terrain data and the noise propagation model. The smartphone equipped with a noise monitoring App provided an opportunity to collect the noise data. Web mapping with free geospatial data offered terrain information. The integration of noise data with terrain data using the noise model can generate noise maps. A smartphone-based technique where users are encouraged to participate in noise monitoring to determine the noise map for their surrounding areas will enable a crowdsourcing technique for mapping.

\section{OBJECTIVES}

The authors here have tried to find out the techniques with which they can measure the extent of noise pollution in city areas using a low cost, and convenient means. Authors will try to find the technique for

1. Use of smartphones with noise measuring applications to capture the noise levels.

2. Use of open-source map to satisfy the need for geospatial data.

3. Use of crowdsourced noise data and open-source map to generate the noise map for an area using noise model.

\section{METHODOLOGY}

The authors have chosen Lucknow city for noise mapping. The city offered different types of traffic environments. It was planned to characterize the traffic noises in different categories and then utilize the data for mapping the noise for surrounding areas. The noise data were collected for 14 locations at different slots (morning afternoon and evening) using a smartphonebased noise measuring application. Further, the mapping is planned to be done using measured noise data. Once the noise data were captured, it was combined with terrain data. The terrain parameters required for noise mapping were extracted from open source terrain data. The extraction of terrain parameters and mapping is conducted using previously developed algorithms by the authors. Due to the lower precision of noise measurement, noise application in the smartphone was also calibrated with a standard noise meter or sound pressure level meter. The calibration values were then applied over the noise data captured through the noise app, to gather accurately measured noise data for an area. The smartphone-based technique for monitoring was planned to work as a user-friendly and convenient means of data collection. The app which has been calibrated with the standard noise meter was used to generate the noise map for three categories of locations (varying in noise character). This was done after measuring the noise levels at 14 locations of Lucknow city with 10 minutes sampling in three slots daily, through the mobile app.

\subsection{Calibration of the Mobile app with SPL}

The literature reviews suggested, that the authors can easily predict using iPhone and flagship phones of Samsung like note8, note9, A30, A50, etc giving better results as compared to other phones with good microphone quality. The app is being made to collect noise data. However, the recorded noise levels may not be accurate from the mobile app, requiring calibration with the standard sound pressure level meter (noise meter). A different technique for calibration can be a mathematical approach and a conventional approach with varying sounds. In the present study, the tonal sounds and the white noise from a good source were played continuously. The uncalibrated smartphone was placed next to the calibrated or the class $1 \mathrm{SPL}$ at a distance of $1 \mathrm{~m}$ away from the source. Most of the smartphones have dynamic operating noise of high amplitude above $80 \mathrm{~dB}(\mathrm{~A})$ and were not taken into account. Continuously varying the tonal sounds were played and recorded using the smartphone and SPL meter. This had provided the variation or changes over the octave bands. The author could predict the variational changes from the data that was made in the app. In the current research, a noise App called NOISECAPTURE was calibrated with the standard sound pressure level meter CESVA SC310.

\subsection{Computation}

Various terrain parameters such as the distance of a receiver point from source, path loss in diffraction, etc are determined and used for computation of noise reduction or attenuations at different locations around the noise sources (i.e., roads). These are used finally to predict the noise levels and display them as a map. Different computations which are been used in calculating the attenuations are as follows:

$$
\begin{gathered}
\text { D.A. }=20 \operatorname{LOG} 10(D)+11 \\
L_{\Sigma}=10 \log _{10}\left(10^{\frac{L_{1}}{10}}+10^{\frac{L_{2}}{10}}+\ldots .+10^{\frac{L_{1}}{10}}\right) \\
\text { B.A }=5.65+66 \mathrm{~N}+244 \mathrm{~N}^{2}+287 \mathrm{~N}^{3}
\end{gathered}
$$

where

$$
\begin{aligned}
& \text { D.A. }=\text { distance attenuation } \\
& \mathrm{L}_{\Sigma}=\text { logarithmic summation } \\
& \text { B.A. }=\text { barrier attenuation } \\
& \mathrm{D}=\text { direct transmission path } \\
& \mathrm{N}=\text { Fresnel number }
\end{aligned}
$$

Also, in this, the authors have computed for many points around the road crossings and building/barriers, and then the map is generated in the ArcGIS platform. Here in this, the barrier attenuation value lies between 0 to 5 , with higher values for larger path loss. The height of the building is being determined using the previous knowledge of the same in the area. The background noise for the area is assumed to be equal to be 30 $\mathrm{dB}(\mathrm{A})$. Thus, during noise mapping, the authors have changed the values of distant points, if the determined value after attenuation was found to be less than $30 \mathrm{~dB}(\mathrm{~A})$.

\section{RESULTS}

In this basically, the calibration of smartphones with standard sound pressure level meter was shown in Table 1 and Table 2 respectively where the tonal sound (having single frequency) was allowed to monitor or measure the noise of different frequencies simultaneously with noise capture and the standard sound pressure level meter or noise meter keeping it at a distance of 1 meter away from the sound source. From the observation, authors found that generally, the 
NOISECAPTURE measures the different tonal sounds at a variation of around 1 to $5 \mathrm{~dB}(\mathrm{~A})$ less than standard noise meter value. These calibrated values were also added on noises of different frequencies to determine the accurate noise data as measured through the smartphone-based NOISECAPTURE app Further, the authors also conducted separate measurements at a few road crossings. Where noise levels were measured using NOISE CAPTURE and standard noise meter. Typical parameters such as N50 and maximum and minimum noise levels were measured through these instruments and were used to ascertain ranges of calibration as given in the following tables.

In Table 1, basically calibration of Polytechnic chauraha data collected using Sound Pressure level meter and Mobile based app is been shown. Here in this the 4 coordinates having different values where both the SPL and Mobile based app are used together to monitor the noise. The difference between the SPL and mobile app is been calculated and then the average is found which show the range of variation of value

\begin{tabular}{|c|c|c|c|c|c|}
\hline \multicolumn{6}{|c|}{ Polytechnic Chauraha } \\
\hline \multicolumn{2}{|c|}{ Coordinate } & \multirow{2}{*}{$\begin{array}{l}\text { N50 } \\
\text { (SPL) }\end{array}$} & \multirow{2}{*}{$\begin{array}{l}\text { M50 } \\
\text { (app) }\end{array}$} & \multirow{2}{*}{$\begin{array}{l}\text { Diff.= } \\
\text { N50- } \\
\text { M50 }\end{array}$} & \multirow[t]{2}{*}{ Range } \\
\hline $\mathrm{X}$ & $\mathrm{Y}$ & & & & \\
\hline $80.9958^{\circ}$ & $26.8731^{\circ}$ & 67.9 & 68.9 & -1 & \multirow[t]{4}{*}{ \pm 4.225} \\
\hline $80.9936^{\circ}$ & $26.8728^{\circ}$ & 78.6 & 68.9 & 9.7 & \\
\hline $81.0005^{\circ}$ & $26.8732^{\circ}$ & 74.0 & 68.9 & 5.1 & \\
\hline $80.9999^{\circ}$ & $26.8738^{\circ}$ & 71.0 & 68.9 & 2.1 & \\
\hline
\end{tabular}

Table 1. Table showing the calibration using the L50 values from the mobile app and SPL.

In Table 2, the maximum value and the minimum value both at the SPL and the mobile app is measured and then the difference in both the instrument is calculated. With the calculated value authors will find the average of difference that gives the marginal average change in the maximum-minimum values. So from the below calculations, the authors have shown the calibration.

\begin{tabular}{|l|l|l|l|}
\hline Location & Source & $\begin{array}{l}\text { Max. } \\
\text { Value }\end{array}$ & $\begin{array}{l}\text { Min. } \\
\text { Value }\end{array}$ \\
\hline Polytechnic & SPL & 83.6 & 64.7 \\
\cline { 2 - 4 } & App & 75.7 & 63.6 \\
\cline { 2 - 4 } & Difference & 7.9 & 1.1 \\
\cline { 2 - 4 } & Average & \multicolumn{2}{|c|}{$\pm \mathbf{4 . 5}$} \\
\hline
\end{tabular}

Table 2. Table showing the calibration using the difference in the maximum and minimum value of SPL and mobile App

Out of 14 locations, authors have chosen the three different noise ambiance that are polytechnic chauraha, Hazrat ganj chauraha, and haniman chauraha. Polytechnic chauraha which comes in high noise zone area comprises a mixed traffic noise pattern. This crossing is merely surrounded by government offices, malls, college, and few shops and the main source of noise are due to the traffic through heavy vehicles and dense traffic that remains throughout the day. Hazratganj which merely comprises of the market area and the main source is surrounding noise and traffic noise. haniman chauraha is ear to residential area and hospitals with a low level of noise throughout the day. Noise ambiance is analyzed for three different slots for three chosen locations. Figure 1 shows the noise map for the Polytechnic morning slot.

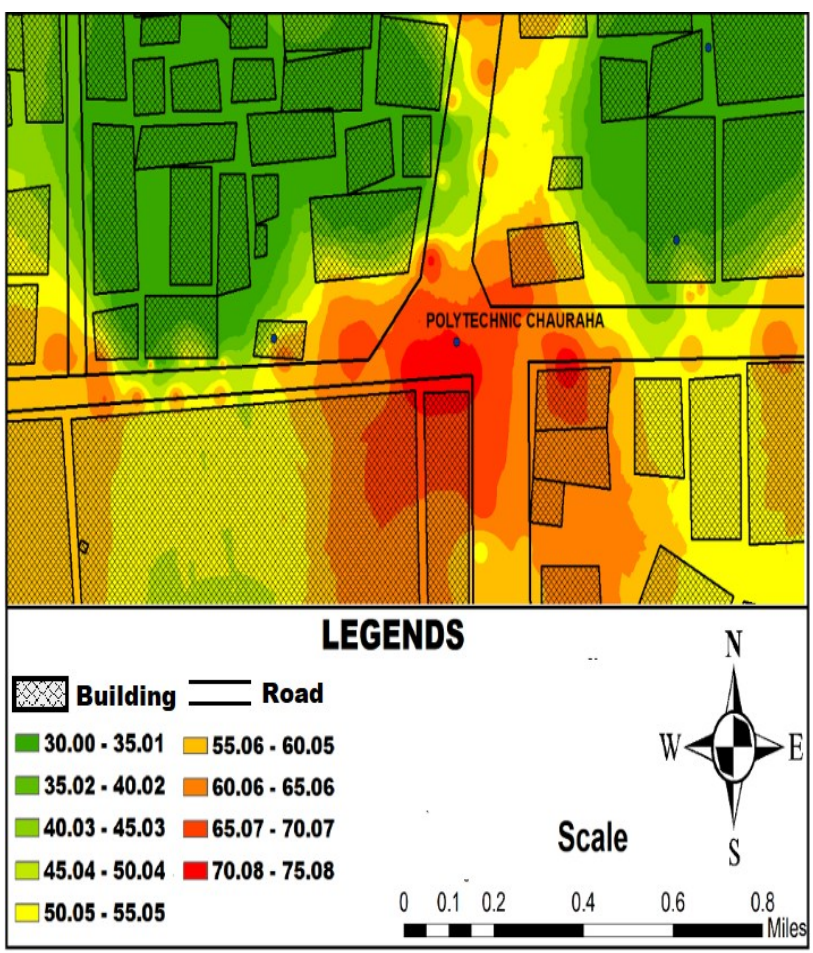

Figure 1. Noise map of Polytechnic Chauraha morning slot with value variation is between 30.00 to 75.08 . Barrier and distance attenuation is also calculated and mapped.

For the same location, the traffic noise variation is analyzed for the afternoon slot which has been shown in Figure 2 with the same parameters

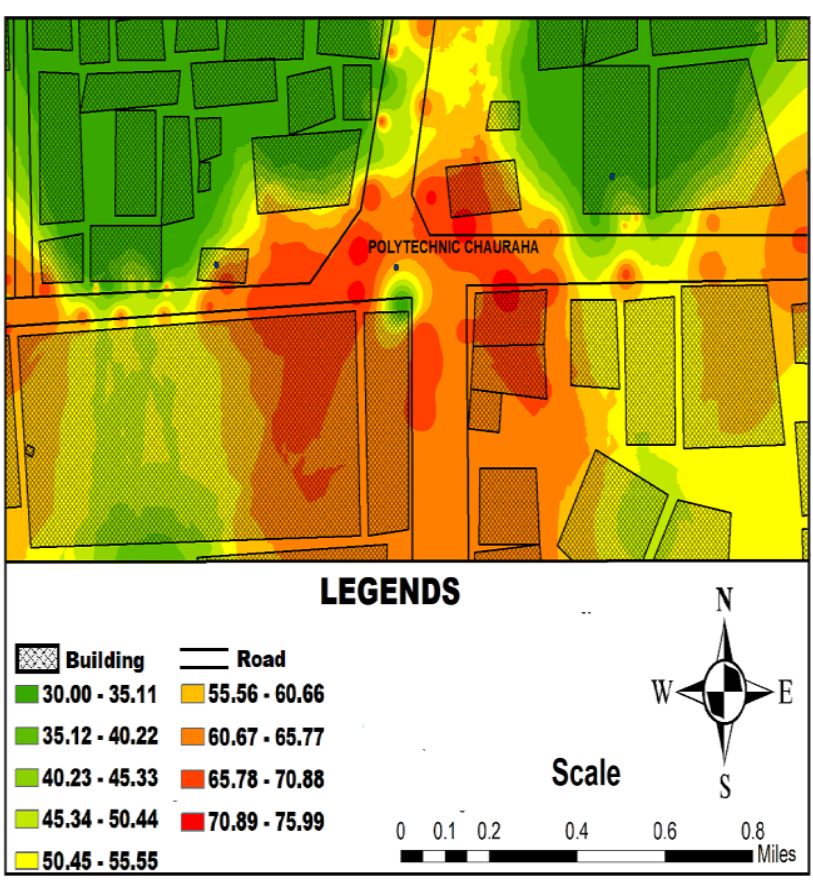

Figure 2. Noise map of Polytechnic Chauraha afternoon slot with value variation is between 30.00 to 75.99 . Barrier and distance attenuation is also calculated and mapped 
For the evening slot, the noise map for Polytechnic Chauraha has shown in Figure 3.

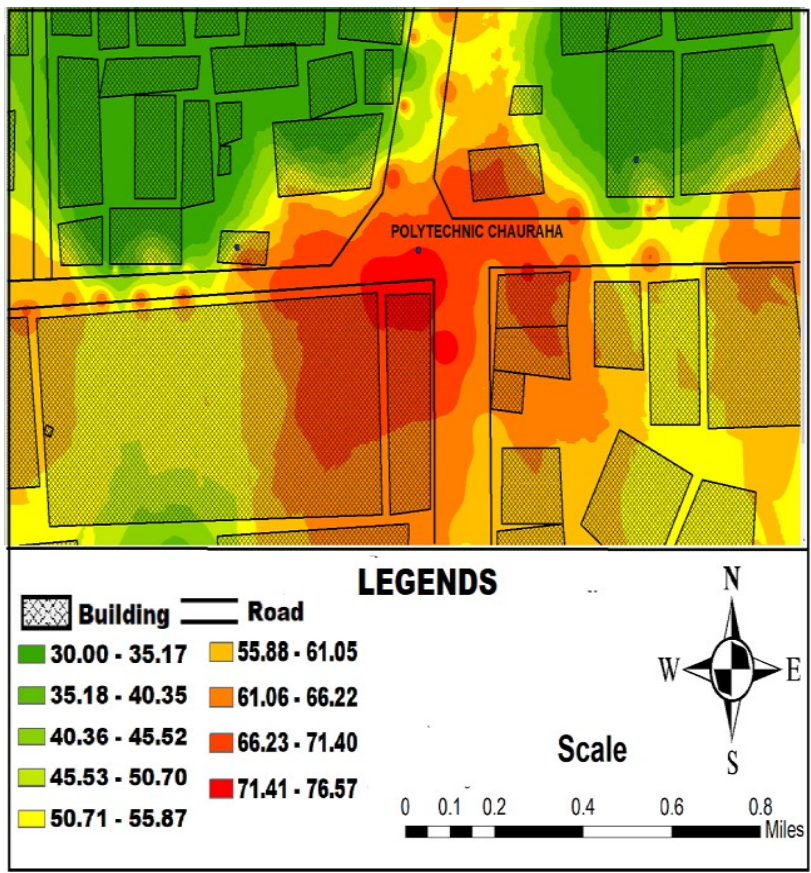

Figure 3. The noise map of Polytechnic Chauraha evening slot with value variation is between 30.00 to 76.57 . Barrier and distance attenuation is also calculated and mapped

Secondly, for Hazratganj Chauraha where the crossing is surrounded by large buildings and complexes. The main source is the vehicles, environmental noise, and market area noise, that remains throughout the day. This comes in a medium noise zone area. Figure 4 shows the noise map for the morning slot.

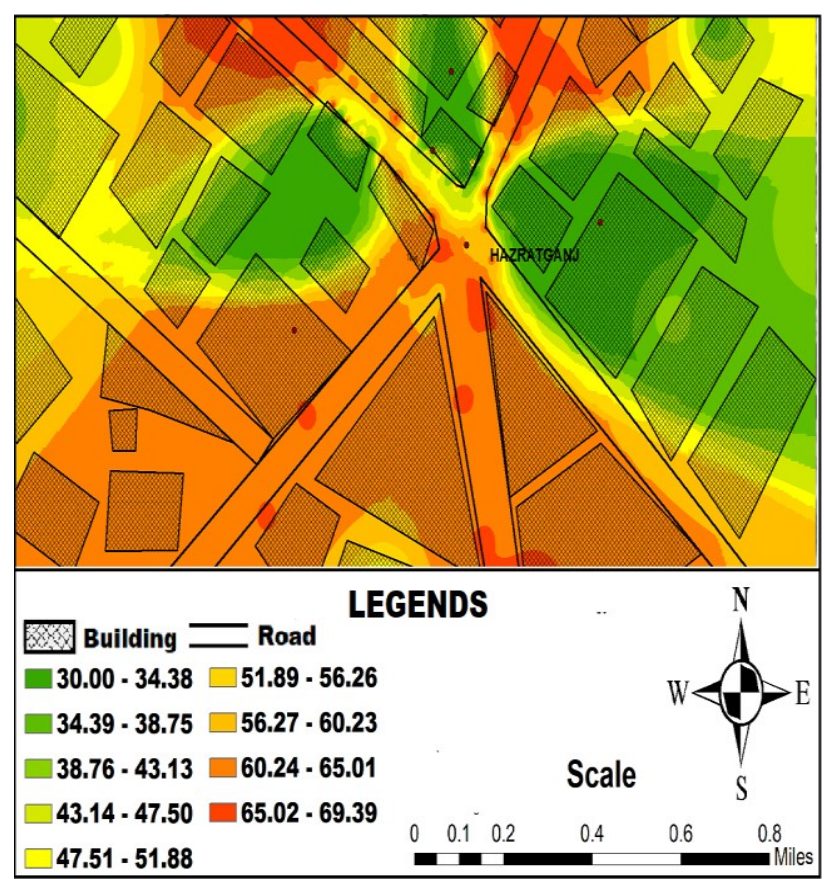

Figure 4. The Noise map of Hazratganj Chauraha of morning slot with value variation is between 30.00 to 69.39 . Barrier and distance attenuation is also calculated and mapped.
The afternoon slot for Hazratganj Chauraha noise map has shown in Figure 5.

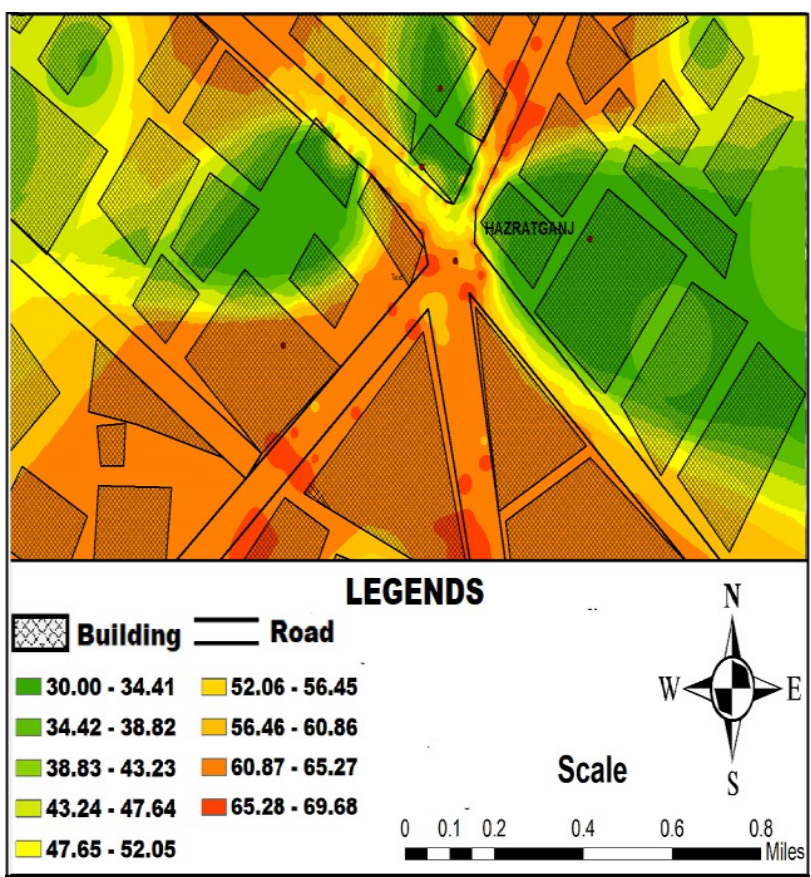

Figure 5. The noise map of Hazratganj Chauraha afternoon slot with value variation is between 30.00 to 69.68 . Barrier and distance attenuation is also calculated and mapped.

For the evening slot, the noise map has shown in Figure 6. The noise values are less as compared to the morning and afternoon slots.

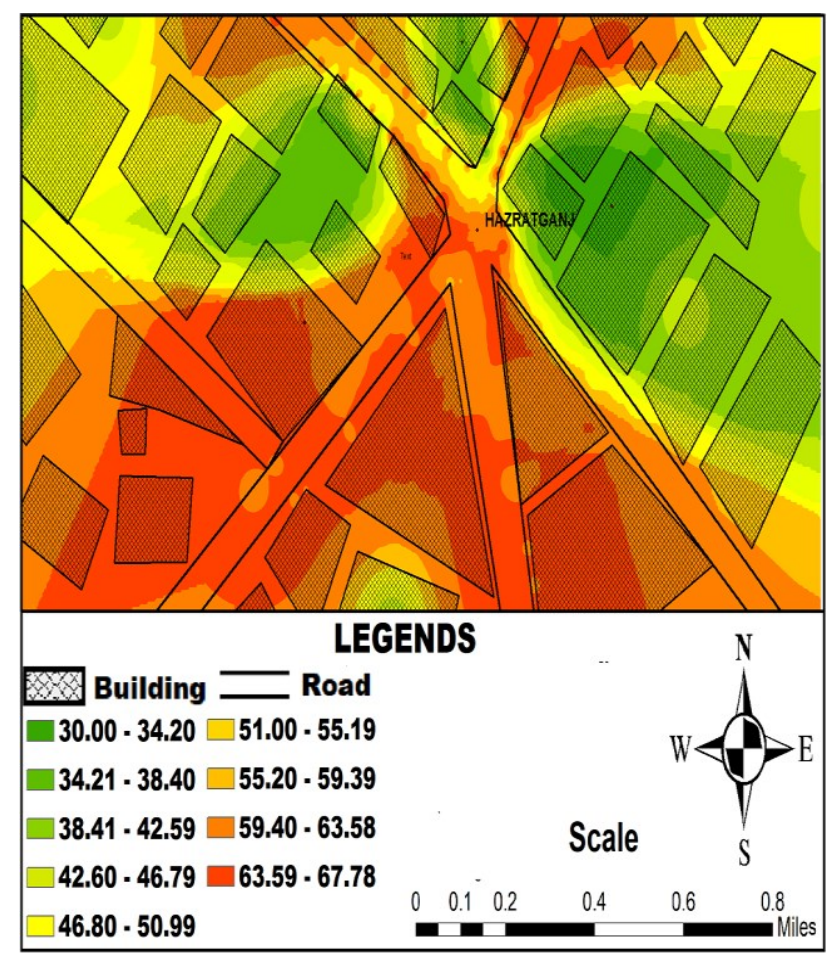

Figure 6. The Noise map of Hazratganj Chauraha evening slot with value variation is between 30.00 to 67.78 . Barrier and distance attenuation is also calculated and mapped. 
The haniman chauraha which is near to the residential area and surrounded by house and hospital and clinics. Here the background noise, environmental noise, and few vehicles over the period are the main source of the noise. Figure 7 shows the noise ambiance for Haniman Chauraha in the morning slot.

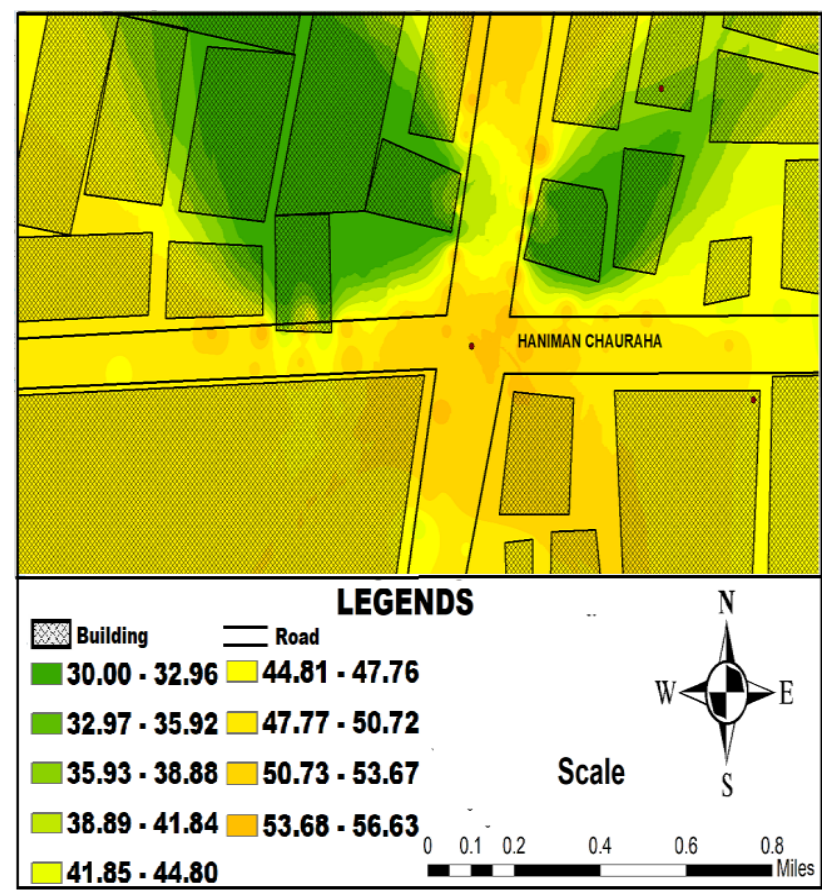

Figure 7. The noise map of Haniman Chauraha of the morning slot with value variation is between 30.00 to 56.63 . Barrier and distance attenuation is also calculated and mapped.

The afternoon slot for Haniman Chauraha noise map has shown in Figure 8.

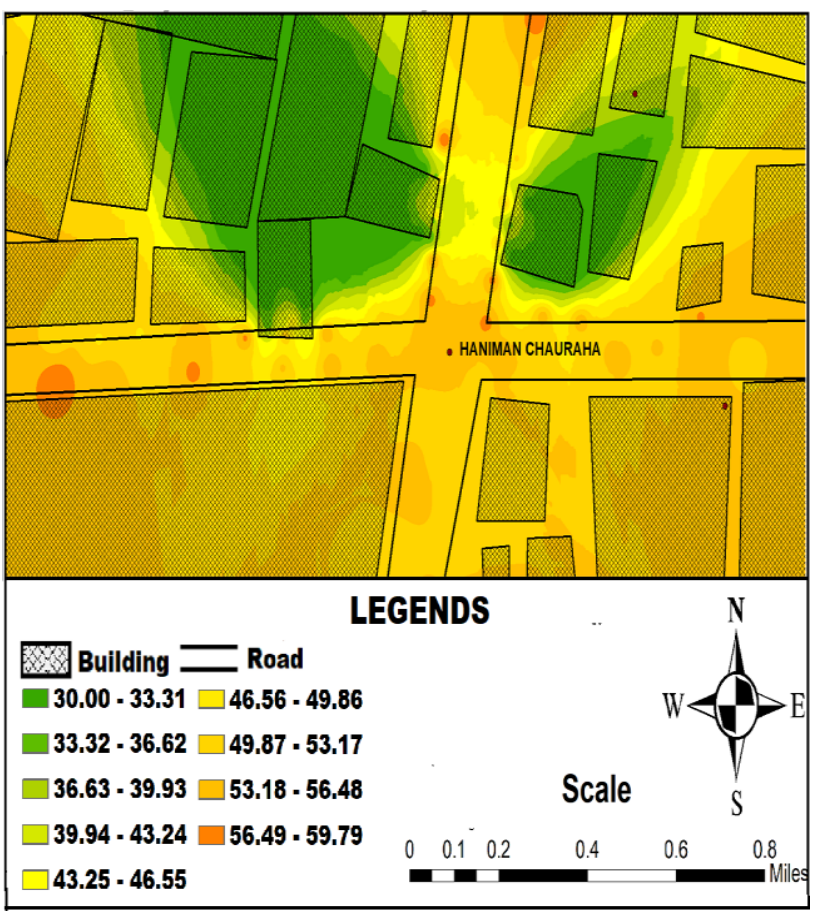

Figure 8. The noise map of Haniman Chauraha of the afternoon slot with value variation is between 30.00 to 59.79. Barrier and distance attenuation is also calculated and mapped.
The evening slot for Haniman Chauraha noise map has shown in Figure 9.

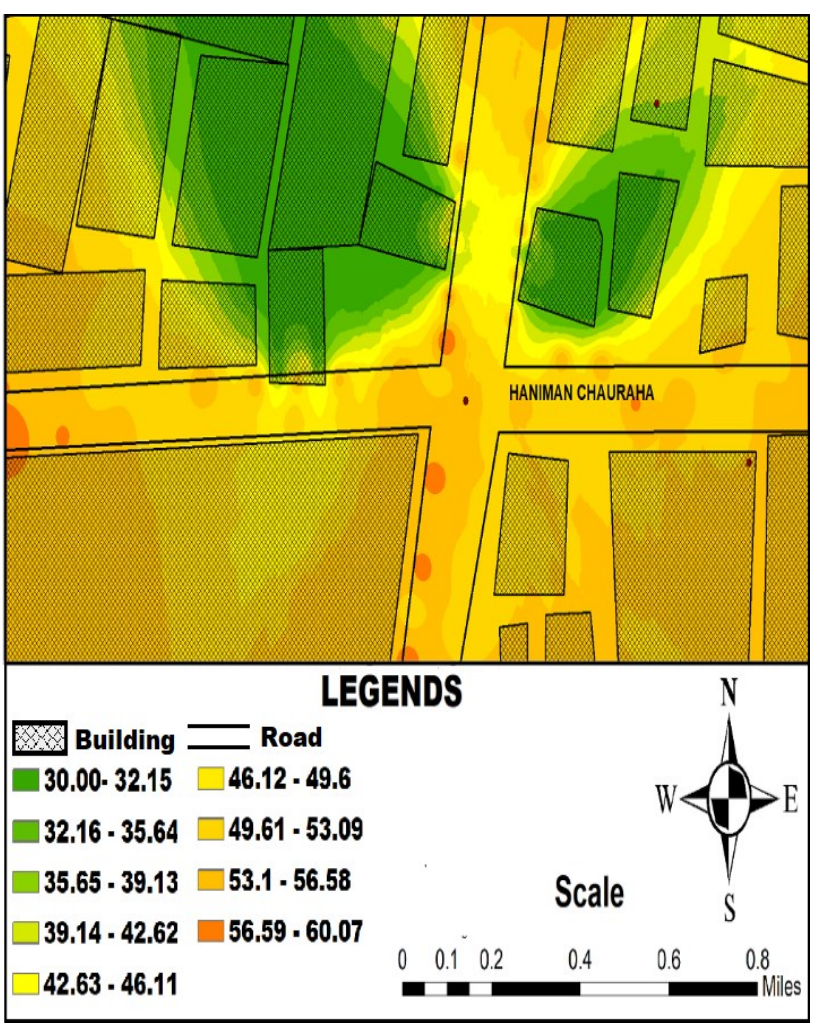

Figure 9. The noise map of Haniman Chauraha of evening slot with value variation is between 30.00 to 60.07 . Barrier and distance attenuation is also calculated and mapped

The three locations Polytechnic Chauraha, Hazratganj Chauraha, and Haniman Chauraha, were also considered based on the noise value that has been measured and also based on recurring survey. By inculcating the prediction model over the noise sources the values over the whole region have been calculated and that provide the variational change in the noise value throughout the day over different slots and the characterized changes can help in determining the level of noise. In the below Table 3, noise ambiance of 14 location has been shown which is the detailed noise survey of the locations that are Hazratganj, Parivartan Chowk, Command Hospital, Loreto Chauraha, Alambagh, Avadh Chauraha, Engineering College, Munshi puliya, Polytechnic Chauraha, IGP Chauraha, Amity Tiraha, Haniman chauraha, Patrakar Puram, and Husadiya Chauraha. The data for the above-mentioned locations have been collected using the NOISE CAPTURE mobile app. Different nomenclature has been used in the table to rectify the problem of knowing the parameters. Here in this M, A, E stands for Morning, Afternoon, and Evening slots respectively. On the other hand L, M, H stands for Low, Medium, and High level of noise that has been monitored. The Leq (Lequivalent) value is measured fro different slots for all the locations and later the average value for the location is calculated that has been shown below. To know the level of exposure throughout the day as sudden a variation in the value may merely affect more over the health aspect of various individuals. From the below values, one can easily predict out that certain locations are having the Leq (Lequivalent) values higher than the permissible exposure limit of the CPCB (central pollution control board, India). People concerning the above crossings complained about impacts various losses like physical, and psychological. 


\begin{tabular}{|c|c|c|c|c|c|}
\hline S.NO & Location & $\begin{array}{l}\text { Type } \\
\text { of } \\
\text { noise }\end{array}$ & $\begin{array}{l}\mathbf{L}_{\mathrm{eq}} \\
(\mathbf{M})\end{array}$ & $\begin{array}{l}\mathbf{L}_{\mathrm{eq}} \\
\text { (A) }\end{array}$ & $\begin{array}{l}\mathbf{L}_{\text {eq }} \\
\text { (E) }\end{array}$ \\
\hline 1 & Hazratganj & M & 69.4 & 67.8 & 69.7 \\
\hline 2 & $\begin{array}{l}\text { Parivartan } \\
\text { Chowk }\end{array}$ & M & 69.8 & 67.4 & 68.4 \\
\hline 3 & $\begin{array}{l}\text { Command } \\
\text { Hospital }\end{array}$ & $\mathrm{H}$ & 75.4 & 69.2 & 73.5 \\
\hline 4 & $\begin{array}{l}\text { Loreto } \\
\text { Chauraha }\end{array}$ & $\mathrm{H}$ & 71.2 & 74.5 & 69.8 \\
\hline 5 & Alambagh & $\mathrm{L}$ & 60.1 & 65.6 & 72.4 \\
\hline 6 & $\begin{array}{l}\text { Avadh } \\
\text { Chauraha }\end{array}$ & M & 69.7 & 65.4 & 72.3 \\
\hline 7 & $\begin{array}{l}\text { Engineering } \\
\text { College }\end{array}$ & $\mathrm{H}$ & 72.7 & 68.4 & 75.1 \\
\hline 8 & $\begin{array}{l}\text { Munshi } \\
\text { Puliya }\end{array}$ & $\mathrm{H}$ & 73.2 & 70.8 & 75.4 \\
\hline 9 & $\begin{array}{l}\text { Polytechnic } \\
\text { Chauraha }\end{array}$ & $\mathrm{H}$ & 75.1 & 76.0 & 76.6 \\
\hline 10 & IGP Chauraha & $\mathrm{H}$ & 72.3 & 69.4 & 74.9 \\
\hline 11 & Amity Tiraha & $\mathrm{L}$ & 62.8 & 60.2 & 64.6 \\
\hline 12 & $\begin{array}{l}\text { Haniman } \\
\text { Chauraha }\end{array}$ & $\mathrm{L}$ & 56.7 & 59.8 & 60.1 \\
\hline 13 & $\begin{array}{l}\text { Patrakar } \\
\text { Puram }\end{array}$ & M & 64.4 & 69.1 & 73.4 \\
\hline 14 & $\begin{array}{l}\text { Husadiya } \\
\text { Chauraha }\end{array}$ & $\mathrm{L}$ & 56.8 & 62.6 & 64.8 \\
\hline
\end{tabular}

Table 3. The Leq values at a different location and the different slot has been shown. Nomenclature H, M, L are high, medium, low-level noise, and here M, A, E stands for the morning, afternoon, and evening.

From the above result authors could know that after calibration of a smartphone with standard sound pressure level meter using the tonal sound, the authors had to do a top-up of $0-4.5 \mathrm{~dB}(\mathrm{~A})$ in different frequency-specific noises to determine the accurate value of noise as measured through NOISECAPTURE. Also, from the map showing a different color pattern, the authors could determine that Polytechnic Chauraha comes in high-risk noise zone area, on the other hand, for medium noise zone area i.e. Hazratganj Chauraha is there and for Haniman Chauraha which comes in low-risk zone area which is showing very less variation in noise level. The mapped noise levels were also compared in the above 3 locations with the values measured through standard noise meter (sound pressure level meter). Generally, for several test sites, the variations were found to be within \pm 3 to $4 \mathrm{~dB}(\mathrm{~A})$. Road traffic noises can have hazardous health implications. People concerning the above crossings complained about annoyance, hypertension, tinnitus, cardiovascular disease, sleep disturbance, etc. This type of problem in human beings impacts various losses like physical, and psychological.

\section{CONCLUSION}

The authors have reviewed the work of various researchers working in noise mapping area. The challenge of noise data monitoring for large areas of cities is tried to be handled with a smartphone-based noise capturing. Authors have tried to predict the noise map using the noise model on a web platform. The approach involved two aspects: easy and effective measurement of traffic noise and creating a noise map of the whole area using limited measurement data. As the authors are moving toward modernization the need for a smart, convenient, and low-cost technique for collection and mapping of noise data has been fulfilled. A smartphone-based noise app has been utilized to limit the need for costly SPL or Noise meter. Also, crowdsourcing has been proposed on the web platforms to accumulate noise data along with terrain data. Extraction of terrain parameters and use of them with noise modeling algorithms will cater to the need for the generation of noise maps over the web environment. The ESRI's ArcGIS offers the opportunity to predict the noise values for different locations in the city environment, and showcase the results as colorful maps in the web platform. The collaborative mapping, and linking that with the health parameters of users will give an idea about hazardous scenarios present at various city crossings. The detailed time, season, and location-specific mapping will inform the users about the imminent danger. If a person needs to work near any red zone of the map for a long duration, then he will be getting greater exposure to noise with high $\mathrm{dB}$ values. Thus, they will have a higher risk of health concerns as compared to the person residing or working near any green zone.

\section{FUTURE SCOPE}

The users will be able to ascertain the likely impacts easily, at a low cost using the mobile phone. Thus, it can be applied to any desired location. People can get detailed information about noise ambiance at a place and relate it to likely noise hazards, unlike conventional practices. The study can also focus on finding the choices of people of different areas for the hospital, school, residential places vis a vis the noise impact to those places. This will give the best or preferred locations for noisesensitive infrastructures. The cue from the above study will guide determining the best locations for setting up a new hospital, school, and residential buildings. As the entire prediction could be made geospatially in a GIS environment, the additional cue of distance from house to the hospital, house to school, etc., the availability of conveyance, the nature of transport, traffic condition, etc. could also be weighted to decide the best or preferred location for noise-sensitive infrastructure.

\section{ACKNOWLEDGEMENT}

Authors would like to acknowledge the support from google especially the google map for providing the authors the access to their resources so that the authors can make the noise map of different places without violating any norms and policies. Also, the authors like to thank the noise capture app using which the authors have collected the data and executed the work. Special thanks to ESRI for helping the authors in mapping with their software.

\section{REFERENCES}

Akintuyi, Akin, Adekunle S Raji, and Emmanuel Wunude. 2014. "GIS-Based Assessment and Mapping of Noise Pollution in Bariga Area of Lagos State, Nigeria GIS-Based Assessment and Mapping of Noise Pollution in Bariga Area Of Lagos State, Nigeria Akintuyi, A. O *; Raji, S. A .** Adewuni, D *. and Wunude, E. O.” (November).

Banerjee, D., S. K. Chakraborty, S. Bhattacharyya, and A. Gangopadhyay. 2008. "Evaluation and Analysis of Road Traffic 
Noise in Asansol, West Bengal." Journal of the Institution of Engineers (India): Environmental Engineering Division 89(SEPT): 9-16.

Biswas, S, and B Lohani. 2008. "Development of HighResolution 3D Sound Propagation Model Using LIDAR Data and Air Photo." The International Archives of the Photogrammetry: 1735-40.

Bocher, E. et al. 2015. "Crowdsourcing of Noise Map Pollution Using Smartphones.” (JUNE).

Bocher, Erwan, et al. 2019. "Noisemodelling: An Open Source GIS-Based Tool to Produce Environmental Noise Maps." ISPRS International Journal of Geo-Information 8(3).

Esri, An. 2018. "ArcGIS Secure Mobile Implementation Patterns." (November).

Maguire, David J. 2016. "ArcGIS: General-Purpose GIS Software." Encyclopedia of GIS: 1-8.

Mofeed, Bayan, Rana Imam, and Ahmad Jamrah. 2013. "Noise Mapping Using GIS: A Case Study from Amman." Journal of American Science 9(12): 646-52.

Münzel, Thomas, et al. 2018. "The Adverse Effects of Environmental Noise Exposure on Oxidative Stress and Cardiovascular Risk." Antioxidants and Redox Signaling 28(9): 873-908.

Tandel, Bhaven, and Dipeshkumar Sonaviya. 2018. “A Quick Review on Noise Propagation Models and Software."” (February): 6. 